\title{
Perinatal Management of Pregnancy Complicated by Autosomal Dominant Emery- Dreifuss Muscular Dystrophy
}

\author{
Megumi Sato, MD, $\mathrm{PhD}^{1}$ Hiromitsu Shirasawa, MD, $\mathrm{PhD}^{1}$ Kenichi Makino, MD, $\mathrm{PhD}^{1}$ \\ Hiroshi Miura, MD, $\mathrm{PhD}^{1}$ Wataru Sato, MD, $\mathrm{PhD}^{1}$ Dai Shimizu, MD, $\mathrm{PhD}^{1}$ Naoki Sato, MD, $\mathrm{PhD}^{1}$ \\ Jin Kumagai, MD, $\mathrm{PhD}^{1} \quad$ Akira Sato, MD, $\mathrm{PhD}^{1} \quad$ Yukihiro Terada, MD, $\mathrm{PhD}^{1}$ \\ ${ }^{1}$ Department of Obstetrics and Gynecology, Akita University Graduate \\ School of Medicine, Akita, Japan \\ Am J Perinatol Rep 2016;6:e145-e147. \\ Address for correspondence Akira Sato, MD, PhD, Department of \\ Obstetrics and Gynecology, Akita University Graduate School of \\ Medicine, 1-1-1 Hondo, Akita, Japan 010-8543 \\ (e-mail: satoa@doc.med.akita-u.ac.jp).
}

\begin{abstract}
Keywords

- Emeri-Dreifuss muscular dystrophy

- preeclampsia

- congestive heart failure

- HELLP syndrome

Introduction Autosomal dominant Emery-Dreifuss muscular dystrophy (AD-EDMD) is rare compared with other forms of muscular dystrophy and is characterized by cardiac conduction defects. Here, we present the case of a patient diagnosed with AD-EDMD during the first trimester of pregnancy who developed acute preeclampsia and subsequently, congestive heart failure (CHF) following cesarean section.

Case A 36-year-old, gravida 0 para 0 woman was diagnosed with AD-EDMD by genetic testing during the first trimester of pregnancy, and she suddenly developed preeclampsia and partial HELLP (hemolytic anemia, elevated liver enzymes, and low platelets) syndrome at 33 weeks of gestation. The patient subsequently developed CHF following cesarean section.

Conclusion CHF can occur as a direct result of the cardiac defects arising due to EDMD, and therefore, careful prenatal and postpartum management is recommended for such cases.
\end{abstract}

Emery-Dreifuss muscular dystrophy (EDMD) is characterized by early contractures, slow progressive muscle wasting, and weakness accompanied by a distinctive humeroperoneal distribution and cardiac conduction defects leading to dilated cardiomyopathy. ${ }^{1}$

Three genes are known to be associated with EDMD: encoding emerin (EMD) and encoding FHL1 (FHL1), which cause X-linked EDMD (XL-EDMD), and encoding lamin A and C (LMNA), which causes autosomal dominant EDMD (AD-EDMD) and autosomal recessive EDMD (AR-EDMD). The diagnosis of XL-EDMD relies upon the failure to detect emerin or FHL1 protein in various tissues and the molecular genetic testing of EMD or FHL1. The diagnosis of AD-EDMD and AR-EDMD also relies upon the molecular genetic testing of LMNA. ${ }^{2}$ The disease frequency is estimated at 1/100,000 for the X-linked form but remains unknown for AD-EDMD. Only a few rare autosomal recessive cases have been reported so far. ${ }^{3}$

received

August 5, 2015 accepted after revision February 19, 2016
DOI http://dx.doi.org/ 10.1055/s-0036-1581058. ISSN 2157-6998.
There is no specific treatment for EDMD. All patients with EDMD should undergo a detailed cardiac investigation and regular cardiology follow-up because death can occur suddenly in such patients. The implantation of a pacemaker or defibrillator in such patients can help save lives by providing a means of early detection of arrhythmias. ${ }^{3}$

Here, we present the case of a patient diagnosed with ADEDMD during the first trimester of pregnancy, who subsequently developed preeclampsia and a partial HELLP (hemolytic anemia, elevated liver enzymes, and low platelets) syndrome during the third trimester and developed postpartum congestive heart failure (CHF) following cesarean section.

\section{Case Report}

A 36-year-old, gravida 0 para 0 woman, with atrial fibrillation and catheter ablation at the age of 33 , visited our hospital to

Copyright $\odot 2016$ by Thieme Medical Publishers, Inc., 333 Seventh Avenue, New York, NY 10001, USA. Tel: +1(212) 584-4662.
License terms

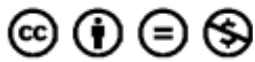


confirm pregnancy. She had first-degree atrioventricular block with junctional rhythm and was admitted to our hospital for 2 weeks for a cardiac examination during the first trimester of pregnancy. After she was discharged from the hospital, she was assessed regularly using electrocardiogram and echocardiography. She had sinus rhythm, and the heart rate during pregnancy was approximately 70 to $80 \mathrm{bpm}$. Genetic testing was performed because of a positive family history of hereditary cardiac disease ( - Fig. 1), and the patient was subsequently diagnosed with AD-EDMD. Her father and uncle, 45 and 40 years of age, respectively, both died of sick sinus syndrome and dilated cardiomyopathy. Her cousin had an implantable cardioverter-defibrillator due to complete atrioventricular block. Moreover, at least five additional family members had been diagnosed with cardiac disorders. The patient and her cousin were found to possess an LMNA gene mutation: c. $673 \mathrm{C}>\mathrm{T}$ (p. Arg225X) that causes AD-EDMD.

The patient was provided regular prenatal care during the initial weeks of pregnancy; normal cardiac function without muscular dystrophy was observed. However, at 33 weeks of gestation, she was found to have high blood pressure and proteinuria without symptoms and was urgently admitted to hospital. Blood pressure at admission was $169 / 105 \mathrm{~mm} \mathrm{Hg}$, and laboratory findings revealed 4+ urinary protein; platelets, 134,000/L; hemoglobin ( $\mathrm{Hb}), 9.4$ $\mathrm{g} / \mathrm{dL}$; lactate dehydrogenase, 377 units/L; aspartate aminotransferase (AST), $84 \mathrm{IU} / \mathrm{L}$; and alanine aminotransferase (ALT), $100 \mathrm{IU} / \mathrm{L}$. Thus, the patient was diagnosed with partial HELLP syndrome. A cesarean section was performed the same day under spinal anesthesia; a baby girl was delivered, weighing 1,549 g, with Apgar scores of 6 and 7 at 1 and 5 minutes, respectively. The baby was transferred to the neonatal intensive care unit because of the prematurity and low birth weight. The maternal blood loss was $692 \mathrm{~g}$ during the surgery and a subsequent $380 \mathrm{~g}$ loss within the first 24 hours of the surgery. Upon admission, nicardipine hydrochloride was administered at $2 \mathrm{mg} /$ hour to help control blood pressure until the postoperative phase, when the treatment was changed to oral $\alpha$-methyldopa at 1,500 $\mathrm{mg} /$ day. Two days postoperatively, the patient complained of dyspnea. A routine chest X-ray revealed an increased cardiothoracic ratio (CTR) at $66.7 \%$ and an ejection fraction (EF) of $62 \%$ on echocardiography. She was diagnosed with $\mathrm{CHF}$, and furosemide was started at $20 \mathrm{mg} / \mathrm{day}$. On the postoperative day 5 , she was transfused with two units of red cell concentrate due to a reduced $\mathrm{Hb}$ level of $6.8 \mathrm{~g} / \mathrm{dL}$. The next day, her $\mathrm{Hb}$ levels elevated to $10.0 \mathrm{~g} / \mathrm{dL}$. In addition, AST was $48 \mathrm{IU} / \mathrm{L}$, ALT was $52 \mathrm{IU} / \mathrm{L}$, and the platelet count was $227,000 / \mathrm{L}$

On the postoperative day 6 , the patient was shifted to the division of cardiovascular medicine in our hospital, and she was able to take oral medicines, including furosemide $20 \mathrm{mg}$, spironolactone $25 \mathrm{mg}$, and $\alpha$-methyldopa $1,500 \mathrm{mg} /$ day. On the postoperative day 16 , the condition of her congestive heart was found to have improved, as CTR was 53\% and EF was $62 \%$. Blood examination revealed $\mathrm{Hb}, 12.0 \mathrm{~g} / \mathrm{dL}$; AST, 27 IU/L; ALT, $40 \mathrm{IU} / \mathrm{L}$; and platelets, 262,000/L. She was discharged from the hospital on the postoperative day 20 . She now has regular follow-up appointments with a cardiologist

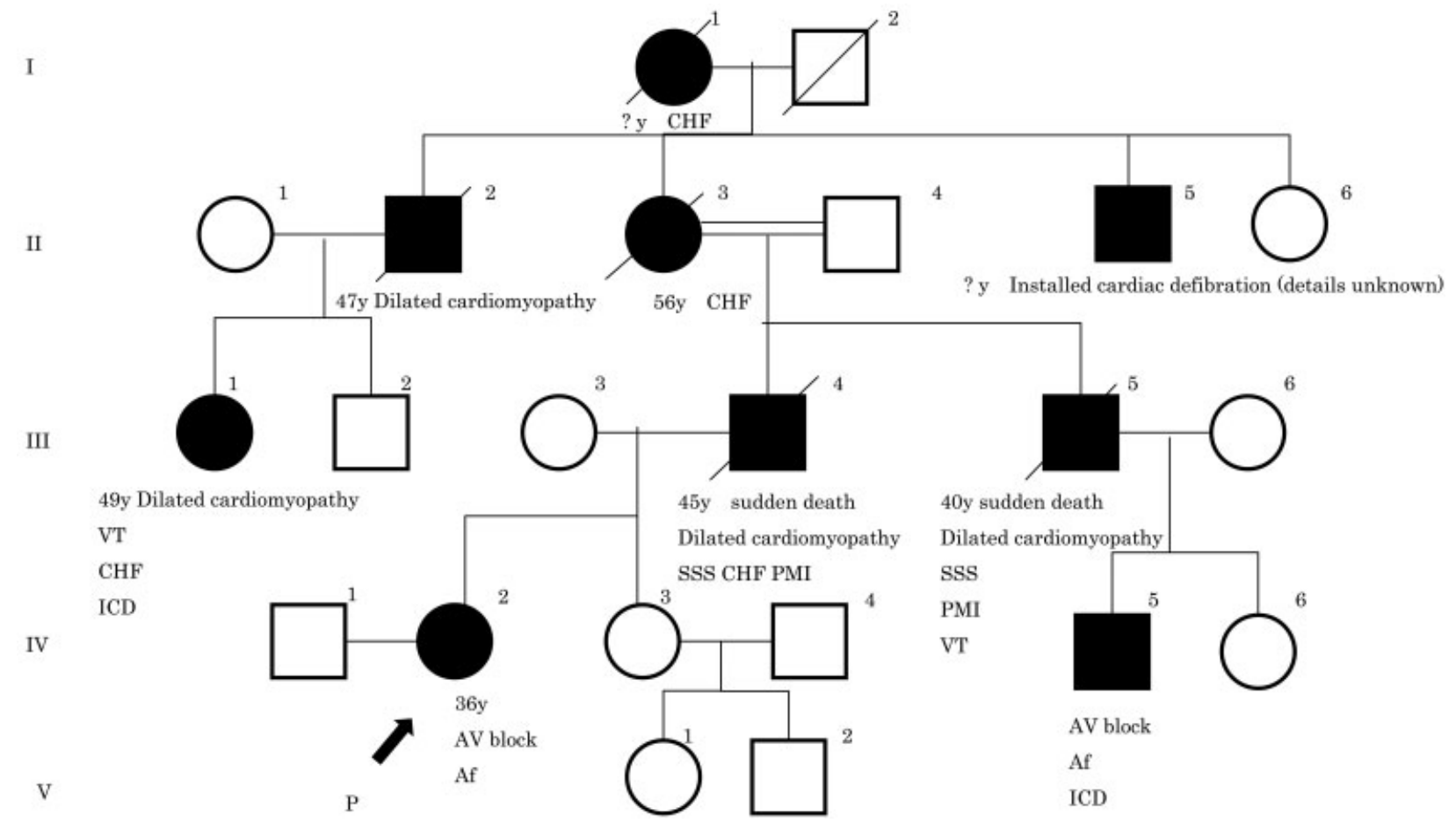

Fig. 1 Family pedigree. Black squares and circles indicate the patients. The proband is indicated by an arrow. I to IV are generation numbers of the pedigree. IV-2, IV-3, and IV-5 were examined for the responsible gene. Af, atrial fibrillation; AV block, atrioventricular block; CHF, congestive heart failure; ICD, implantable cardioverter-defibrillator; P, probad; PMI, pacemaker implantation; SSS, sick sinus syndrome; VT, ventricular tachycardia. 
and has not had a recurrence of CHF due to dilated cardiomyopathy.

\section{Discussion}

The case presented herein is the first report of pregnancy in a patient with AD-EDMD complicated by severe preeclampsia.

Compared with Duchenne or Becker muscular dystrophy, EDMD is a rare condition. The cardiac conduction defects leading to dilated cardiomyopathy are a typical characteristic of EDMD. ${ }^{1,4}$ These cardiac characteristics may represent the earliest presentation of EDMD and may limit the development of skeletal findings, particularly if there is early sudden death. ${ }^{4}$ This patient had a clinical history of atrial fibrillation before pregnancy, without any underlying skeletal dysfunction. As a genetic mutation was found in the patient and her cousin, it is likely that their cardiac dysfunction will be progressive.

At 33 weeks of gestation, the patient developed the partial HELLP syndrome. Her blood pressure, elevated acutely, and the decision to carry out a cesarean section was made. Nevertheless, she postoperatively developed CHF. In this case, CHF occurred due to the physiological increase in plasma volume during pregnancy, coupled with fluid administration during the surgery. As the patient had a cardiac conduction disorder, her heart rate was not able to increase to compensate. HELLP syndrome is a severe form of preeclampsia comprising mainly vascular spasm and intravascular coagulation. CHF occurs in pregnant patients with EDMD who are predisposed to dilated cardiomyopathy as a result of vascular spasm in HELLP syndrome and increased circulating plasma volume. Therefore, these cases require more careful treatment, such as the management of infusion in situations involving rapid hemodynamic changes, that is, HELLP syndrome and cesarean section. The present case clearly highlights the need for careful attention during the clinical management of such patients.

In women affected with preeclampsia, the risk of subsequent cardiovascular and cerebrovascular diseases is doubled compared with age-matched controls. Preeclampsia with severe features is strongly associated with the risk of adverse cardiovascular outcomes in the future. ${ }^{5}$ Melchiorre et al reported that women with preeclampsia are at a significantly higher risk of developing asymptomatic LV dysfunction within 1 to 2 years of delivery. ${ }^{6}$ As with the current case, severe preeclampsia may precipitate cardiovascular diseases in patients with EDMD. Consequently, these patients require lifelong treatment and observation.
The present patient developed hereditary EDMD during the first trimester of pregnancy. The age of onset, severity, and progression of muscular and cardiac involvement are known to demonstrate both inter- and intrafamilial variability in EDMD. ${ }^{7}$ In this particular family, the onset was in the third decade of life, advanced over the next 10 years, and death occurred in the fourth decade. Pregnancy complicated by ADEDMD is rare as manifestations are not typically present at the maternal age, and skeletal findings are unremarkable. However, we predict that the number of such cases will increase over time due to the advancing maternal age. Physicians are, therefore, required to take sufficient family history upon the first presentation of such patients. If necessary, we should also consider genetic counseling, which may lead to the diagnosis of familial disorders. It may become possible to correspond in advance to different conditions which may occur.

In conclusion, cardiac disorders in patients with ADEDMD are progressive. Consequently, pregnant women complicated by AD-EDMD should be carefully treated during the peripartum and perioperative period. The number of such patients will increase in the future because of the advancing maternal age in developed countries. Therefore, it is necessary to assess cardiac function regularly not only during the peripartum phase, but also during the remainder of the patient's life.

\section{References}

1 Helbling-Leclerc A, Bonne G, Schwartz K. Emery-Dreifuss muscular dystrophy. Eur J Hum Genet 2002;10(3):157-161

2 Bonne G, Leturcq F, Ben Yaou R. Emery-Dreifuss muscular dystrophy. In: Pagon RA, Adam MP, Ardinger HH, et al, eds. GeneReviews [Internet]. Seattle, WA: University of Washington, Seattle; 19932016. Available at: http://www.ncbi.nlm.nih.gov/books/NBK1436/. Accessed March 10, 2016

3 Raffaele Di Barletta M, Ricci E, Galluzzi G, et al. Different mutations in the LMNA gene cause autosomal dominant and autosomal recessive Emery-Dreifuss muscular dystrophy. Am J Hum Genet 2000;66(4):1407-1412

4 Puckelwartz M, McNally EM. Emery-Dreifuss muscular dystrophy. Handb Clin Neurol 2011;101:155-166

5 Young BC, Levine RJ, Karumanchi SA. Pathogenesis of preeclampsia. Annu Rev Pathol 2010;5:173-192

6 Melchiorre K, Sutherland GR, Liberati M, Thilaganathan B. Preeclampsia is associated with persistent postpartum cardiovascular impairment. Hypertension 2011;58(4):709-715

7 Carboni N, Porcu M, Mura M, et al. Evolution of the phenotype in a family with an LMNA gene mutation presenting with isolated cardiac involvement. Muscle Nerve 2010;41(1):85-91 